\title{
Penetration von Pollenallergenen durch die Haut - Entwicklung einer neuen Nachweismethode
}

\author{
U. Jacobi \\ K. Wilken \\ S. Tribius \\ H. Schaefer \\ W. Sterry \\ J. Lademann \\ M. Worm
}

Penetration of Pollen Allergens in the Skin - Development of a New Proof Method

\section{Zusammenfassung}

Voraussetzung für die Entstehung IgE-vermittelter Reaktionen der Haut ist die Fähigkeit von Typ-I-Allergenen, in die Haut einzudringen. Daher war das Ziel der vorgestellten Untersuchungen die Entwicklung einer Nachweismethode zur Penetration von Pollenallergenen in die Haut. Voraussetzung hierfür ist zunächst der Nachweis der Freisetzung von Allergenen aus Pollen bei Kontakt mit Flüssigkeiten, wie z.B. Schweiß oder Wasser. Anschließend wurden modellhaft Lieschgras-Allergene mit Fluoresceinisothiocyanat (FITC) markiert. Nach Applikation dieser Allergene wurde das Stratum corneum (SC) mittels der Abrissmethode entfernt. Diese Methode ermöglicht eine nicht-invasive Entfernung des SC durch das wiederholte Aufbringen und Abreißen von Klebefilmstreifen. Die Untersuchungen wurden exemplarisch am Modell der Schweinehaut durchgeführt. Es gelang erstmals mittels der Fluoreszenz-Mikroskopie, die FITC-markierten Allergene bzw. ihre Fragmente auf allen Abrissen, d.h. im gesamten SC, nachzuweisen. Die Ergebnisse zeigen, dass selbst hoch-molekulare Antigene wie Typ-I-Allergene konzentrationsabhängig in das SC gelangen können. Somit kann der Kontakt von Pollen mit Schweiß auf der Haut zur Penetration von Allergenen in die Haut und damit zur Auslösung der Hautreaktion führen.

\section{Abstract}

The penetration of type I allergens into the skin is necessary for the development for IgE associated skin reactions. The development of a method to study the penetration of pollen allergens into the skin was the aim of the present study. These studies are based on the observation that a release of allergens from pollen was determined after the contact with sweat or water. Next, the allergens of grass pollen were labeled with fluorescein isothiocyanate (FITC). After application of these FITC-labeled allergens, the Stratum corneum (SC) was removed using the tape stripping procedure. This method allows to remove the SC completely by a successive application and stripping of an adhesive tape. These investigations were performed in vitro on porcine skin. Finally, the FITC-labeled allergens or their fragments were detected using fluorescence microscopy on all tape strips taken (up to 50) reflecting the complete SC. This result indicates the ability of large molecules as type I allergens to penetrate into the skin depending on their concentration. Therefore, the contact of pollen with sweat on the skin may lead to the penetration of the allergens into the skin which represents the basis for eliciting a skin reaction by type I allergens.

\section{Einleitung}

Die Zahl allergischer Erkrankungen wie das allergisches Asthma oder die atopische Dermatitis hat in den letzten Jahren zugenommen. Dabei nehmen Erkrankungen durch in der Luft enthaltene Typ-I-Allergene (Aeroallergene) wie z.B. Pflanzenpollen ei- nen wesentlichen Platz ein. Die Zahl pollenassoziierter allergischer Erkrankungen hat sich in Deutschland und anderen Industrienationen in den letzten 10 Jahren verdoppelt [1,2]. Zu diesen Allergien zählt ebenfalls die im Gesundheitswesen oft auftretende Latex-Allergie, die durch den Kontakt mit Proteinen der Hevea brasiliensis ausgelöst wird [3]. Neben den klassischen

Institutsangaben

Klinik für Dermatologie, Venerologie und Allergologie, Medizinische Fakultät Charité,

Humboldt-Universität Berlin

Korrespondenzadresse

J. Lademann · Klinik für Dermatologie, Venerologie und Allergologie · Medizinische Fakultät Charité ·

Humboldt-Universität zu Berlin · 10098 Berlin·E-mail: juergen.lademann@charite.de

Bibliografie

Akt Dermatol 2003; 29: 202-205 @ Georg Thieme Verlag Stuttgart · New York · ISSN 0340-2541 
Typ-I-Allergien existieren auch Erkrankungen, die durch TypI-Allergene getriggert oder verschlechtert werden. Dies ist insbesondere bei der atopischen Dermatitis zu beobachten, an der bis zu 15\% der Bevölkerung in Europa leiden [4]. Die Zunahme atopischer Erkrankungen ist von berufsdermatologischer Relevanz, da zumindest die bestehende atopische Dermatitis einen wichtigen, prädisponierenden Faktor für das Entstehen berufsbedingter Ekzeme, z. B. im Friseurberuf, darstellt.

Allergene können nicht nur über die Schleimhaut als Folge von Inhalation, sondern auch perkutan über die Haut aufgenommen werden $[5,6]$. Diese Beobachtung basiert auf klinischen Phänomenen wie Erythemen und Ödemen nach Reibetests mit Pflanzen oder Früchten [7] oder dem Auftreten von ekzematösen Hautveränderungen durch die Anwendung des „Atopie-PatchTests" [8-10]. Viele Patienten mit atopischer Dermatitis entwickeln ihre Hautveränderungen ausschließlich im Bereich der luftexponierten Areale (Abb. 1) [11].

Zwei Prozesse spielen bei der Entstehung Typ-I-allergischer Erkrankungen eine Rolle, zum einen die Sensibilisierungs- und zum anderen die Induktionsphase [12,13]. Beide Phasen können

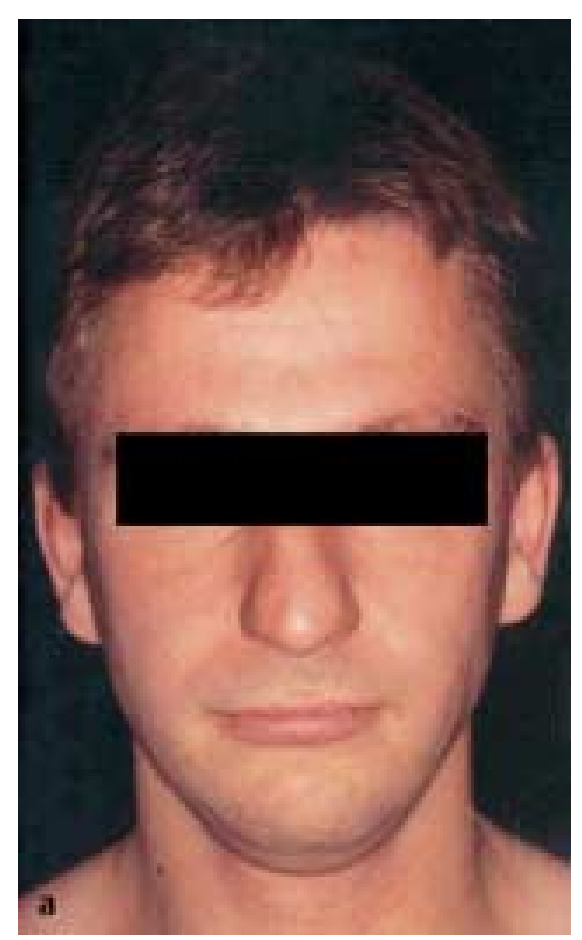

Abb. 1 Typische Lokalisation der durch Aeroallergene getriggerten, atopischen Dermatitis im Bereich der luftexponierten Hautareale. a Gesicht, b Dekolleté.

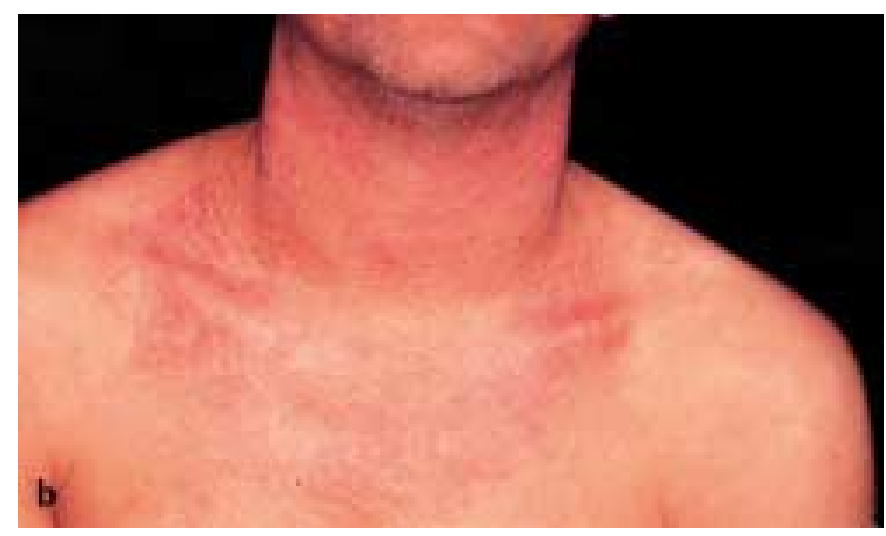

durch Hautkontakt mit den Allergenen hervorgerufen werden. Hierbei kommt der Penetration der Allergene in und durch das Stratum corneum (SC) eine Schlüsselrolle zu. Während die Barriere der gesunden Haut das Eindringen von Partikeln wie Pollenallergenen meist verhindert, geht man bei der atopischen Dermatitis davon aus, dass Mikrotraumata vorliegen, durch die ein verändertes Penetrationsverhalten ermöglicht wird [14,15]. In jüngster Zeit wird auch den Hautanhangsgebilden, insbesondere den Haarfollikeln, als möglichen Penetrationswegen eine Bedeutung zugeschrieben [16].

Das therapeutische Vorgehen der durch Allergene getriggerten, atopischen Dermatitis beinhaltet derzeit die Anwendung symptomatisch wirksamer Medikamente. Hierzu gehören vor allem topisch applizierte, antiinflammatorisch wirksame, steroidhaltige oder immun-modulatorisch wirksame Externa wie Tacrolimus oder Pimecrolimus. In ausgeprägten Fällen werden auch immunsuppressive Substanzen wie z.B. Cyclosporin A eingesetzt. Eine, an den Ursachen der Erkrankung angreifende Therapie steht bis heute nicht zur Verfügung.

Aus diesem Grund konzentrieren sich Untersuchungen, die derzeit an der Hautklinik der Charité durchgeführt werden, auf den bisher nicht geklärten Mechanismus der Penetration von Pollenallergenen in und durch die Haut. Es wurden für die Auslösung der atopischen Dermatitis relevante Typ-I-Allergene als Modell für die Untersuchungen ausgewählt. Zunächst wurde untersucht, wie sich Pollen nach Zugabe von Schweiß und Wasser verhalten. Die beobachtete Freisetzung der Allergene war die Basis, um die Penetration dieser Proteingemische mit Molekülgrößen zwischen 20 und $70 \mathrm{kDa}$ zu untersuchen.

Zu diesem Zweck mussten neue Methoden entwickelt werden. Die Markierung der Allergene mit Fluorescein-isothiocyanat (FITC) ermöglichte ihre Detektion im Stratum corneum von Schweineohren, die ein geeignetes Modell für die humane Haut darstellen [17]. Die Abriss-Methode, bei der das SC vollständig und nicht-invasiv durch wiederholtes Aufkleben und Abreißen von Klebefilmstreifen entfernt wird [18], ermöglicht in Kombination mit der Fluoreszenzmikroskopie die Detektion der FITCmarkierten Allergene. Auf den erhaltenen Ergebnissen aufbauend sollen zukünftig Präventionsmaßnahmen zur Verhinderung der Penetration von Allergenen in die Haut entwickelt werden.

\section{Material und Methoden}

\section{Freisetzung der Allergene}

Gesammelte Kiefer-Pollen wurden auf einem Objektträger mit 5 Tropfen humanem Schweiß bzw. dest. Wasser versetzt. Die Pollen wurden zuvor und danach mittels eines Mikroskops (SystemMikroskop BX60, Olympus Optical Co., Hamburg) fotografiert, das mit einer Digitalkamera (SIS ColorView12, Soft Imaging System $\mathrm{GmbH}$, Münster) ausgerüstet war.

\section{Markierung von Allergenen mit FITC}

Der Lieschgraspollen-Extrakt (lyophilisiert, 450000 SQ-Einheiten, Fa. ALK Scherax, Hamburg) wurde in PBS-Puffer (SigmaAldrich Chemie GmbH, Deisenhofen) mit einem 50fachen Überschuss an Fluorescein-isothiocyanat (FITC, Sigma-Aldrich Che- 
mie $\mathrm{GmbH}$, Deisenhofen) über $2 \mathrm{~h}$ bei $4^{\circ} \mathrm{C}$ inkubiert. Das überschüssige FITC wurde anschließend durch die Zugabe von L-Alanyl-L-Glutamin (Dipeptamin ${ }^{\circledR}$, Frisenius Kabi Deutschland $\mathrm{GmbH}$, Bad Homburg) gebunden. Die Abtrennung der FITC-markierten Allergene erfolgte mittels Membrandialyse (Spectra/ Por 1, MWCO 6-8000, Theodor Karow GmbH, Berlin) über $92 \mathrm{~h}$ unter Lichtkarenz. Das Dialysat (PBS-Puffer) wurde regelmäßig gewechselt, bis sich ausschließlich FITC-markierte Allergene in der Testlösung befanden. Die FITC-markierte Allergenlösung und die Waschlösung wurden aliquotiert und bei $-20^{\circ} \mathrm{C}$ aufbewahrt.

\section{Applikation und Nachweis der FITC-markierten Allergene}

Die Lösung der FITC-markierten Allergene (45000 SQ-Einheiten in $20 \mu \mathrm{l}$ ) wurde auf ungebrühte Ohren frisch geschlachteter Schweine auf ein Hautareal von $2 \times 2 \mathrm{~cm}^{2}$ aufgetragen. Zum Vergleich wurden $20 \mu \mathrm{l}$ der Waschlösung auf ein ebenfalls $2 \times 2 \mathrm{~cm}^{2}$ großes Hautareal appliziert. Nach einer Einwirkzeit von 45 min unter Lichtabschirmung wurde das Stratum corneum mittels der Abriss-Methode [18] entfernt. Hierfür wurde ein tesa-Filmstreifen auf das behandelte Hautareal geklebt, mit einer Rolle angedrückt und mit einem schnellen Ruck entfernt. Diese Prozedur wurde auf demselben Hautareal mit einem zweiten tesa-Filmstreifen wiederholt. Insgesamt wurden 50 Abrisse genommen und so das gesamte SC entfernt. Die Abrisse wurden einzeln unter einem Fluoreszenzmikroskop bezüglich der Belegungsdichte mit Korneozyten und der FITC-markierten Allergenproteine im Vergleich zur Kontrolle ausgewertet.

\section{Ergebnisse}

\section{Freisetzung von Allergenen aus Pollen unter Einwirkung von Schweiß bzw. Wasser}

Die Untersuchungen konzentrierten sich zunächst auf die Frage, ob Allergene aus Pollen freigesetzt werden können, wenn diese auf die Haut gelangen. Der Kontakt mit wässrigen Lösungen wie Schweiß könnte hierfür eine Möglichkeit sein. Die durchgeführten Experimente zeigten, dass Pollenkörner tatsächlich bei Kontakt mit humanem Schweiß aufquollen und anschließend unter Freisetzung ihres Inhaltes, der Protein-Konglomerate, platzten (Abb. 2). Das gleiche Verhalten wurde bei Zusatz von Wasser unter dem Lichtmikroskop beobachtet.

\section{Nachweis von Pollenallergenen im SC}

Die freigesetzten Allergene wurden anschließend mittels FITC markiert, um die Penetration in das SC zu untersuchen. Mittels Fluoreszenzmikroskopie wurde für verschiedene Tiefen des SC die Fluoreszenz der FITC-markierten Typ-I-Allergene bestimmt. Dabei zeigte sich, dass die markierten Proteine über die gesamte Dicke des SC nachweisbar waren. Sie waren immer mit der Anwesenheit von Korneozyten assoziiert (Abb.3). Die Fluoreszenz war um die Zellen oder auf deren Oberfläche lokalisiert. Der größte Teil der FITC-markierten Typ-I-Allergene verblieb jedoch auf der Oberfläche und im oberen Bereich des SC.

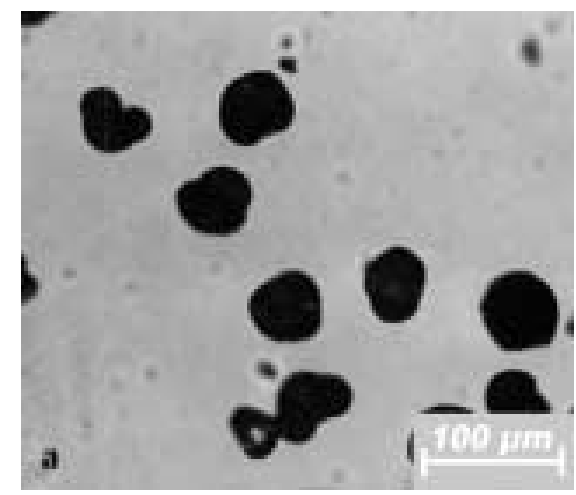

Abb. 2 Pollen vor und nach Kontakt mit menschlichem Schweiß. a Pollen vor Kontakt mit Schweiß, b Pollen nach Kontakt mit Schweiß (im Überblick), c Pollen nach Kontakt mit Schweiß (im Detail).
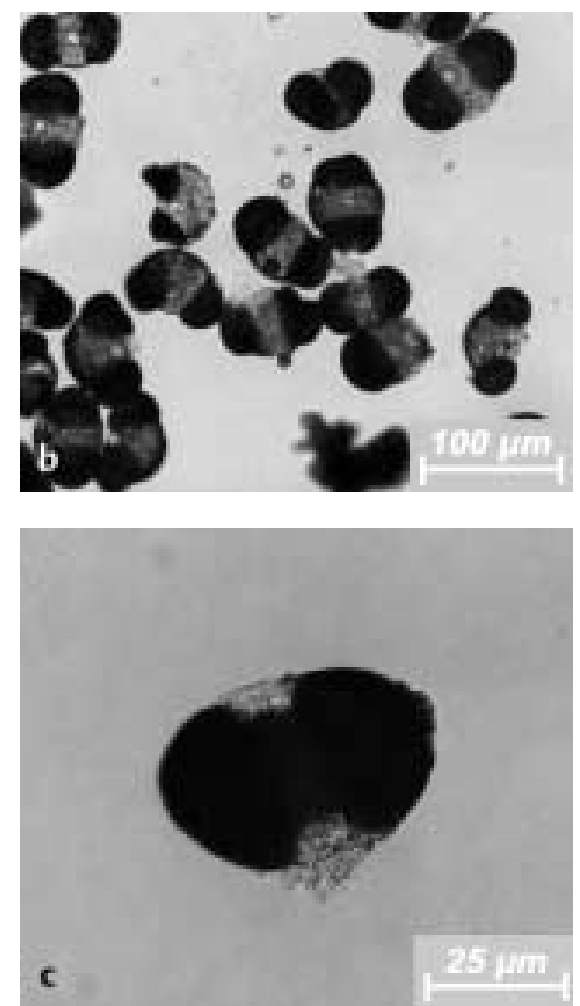

\section{Diskussion}

Die durchgeführten Untersuchungen zeigen, dass der Kontakt von Pflanzenpollen mit humanem Schweiß bzw. Wasser zur Freisetzung des Polleninhaltes führt. Unter diesen Bedingungen werden die Allergene sowohl von der Pollenoberfläche gelöst als auch aus dem Polleninneren freigesetzt und stehen für die Penetration in die Haut zur Verfügung. Somit können Umwelteinflüsse wie Regen oder Transpiration die Auslösung der allergischen Reaktion begünstigen. Demnach bietet auch Kleidung allein unter bestimmten Bedingungen keinen optimalen Schutz vor der Penetration von Pollen in die Haut. So könnte z.B. im Sommer die Transpiration unter eng anliegender, einlagiger Kleidung bei Bestehen einer entsprechenden Sensibilisierung zur Auslösung einer atopischen Dermatitis führen.

Die vorgestellte, neu entwickelte Technik der Fluoreszenzmarkierung in Kombination mit der mikroskopischen Auswertung von Abrissen ermöglicht erstmals die nicht-invasive Untersuchung der Penetration von Typ-I-Allergien auslösenden Proteinen in ihrem nahezu nativen Zustand. Dadurch werden im Er- 

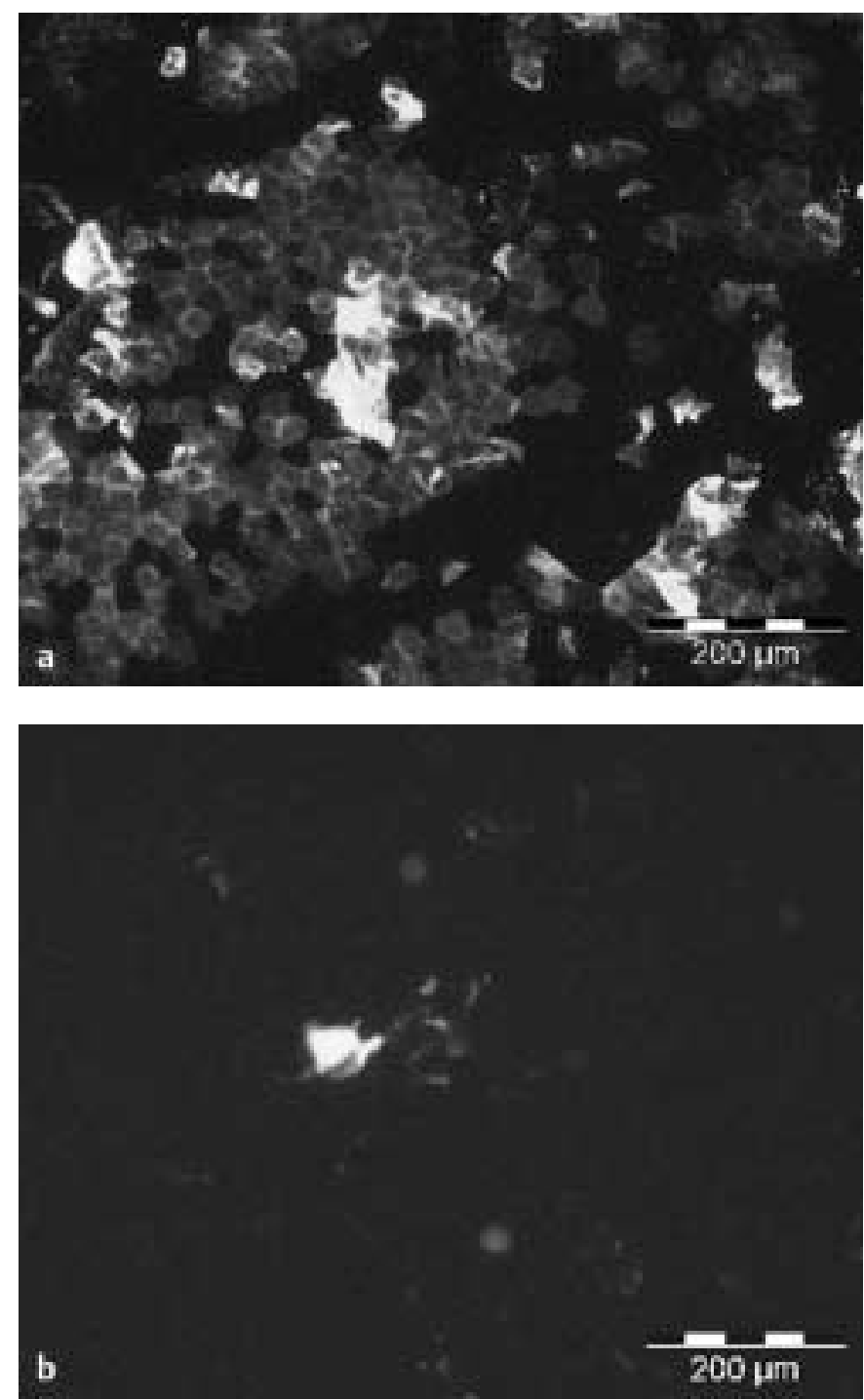

Abb. 3 Fluoreszenzbilder von Abrissen aus verschiedenen Tiefen des SC nach Auftragung FITC-markierter Typ-1-Allergene. a 1. Abriss, b 25 . Abriss.

gebnis auch mögliche Veränderungen in der dreidimensionalen Struktur der Allergene (Proteinfaltung bzw. -entfaltung) während der Penetration oder die Zersetzung durch Enzyme bzw. die Mikroflora miterfasst. So wurde gezeigt, dass die Pollenallergene bzw. ihre Fragmente interzellulär durch das SC penetrieren können. Dieses Ergebnis steht in Übereinstimmung mit Beobachtungen von Ogiso et al. [19,20], die kugelförmige FITC-Dextrane als Modellverbindungen für Proteine eingesetzt haben, um deren Penetration durch die Haut von Ratten zu verfolgen. Die Dextrane penetrierten in Abhängigkeit von ihrer Größe ebenfalls über die Lipidschichten durch das SC.

Diese Ergebnisse stellen die Grundlage für zukünftige Untersuchungen dar, bei denen der Anteil der follikulären Penetration von Pollenallergenen im Mittelpunkt stehen wird.

\section{Literatur}

${ }^{1}$ D'Amato G, Spieksma FT, Liccardi G, Jager S, Russo M, Kontou-Fili K, Wuthrich B, Bonini S. Pollen releated allergy in Europe. Allergy 1998; 53: $567-578$

${ }^{2}$ Beasley R, Keil U, Mutius E, Pearce N. Worldwide variation in the prevalence of symptoms of asthma, allergic rhinoconjunctivitis, and atopic eczema: ISAAC. Lancet 1998; 351: 1225-1232

${ }^{3}$ Sussman GL, Beezhold DH, Kurup VP. Allergens and natural rubber proteins. J Allergy Clin Immun 2002; 110 (2 Suppl): 33 - 39

${ }^{4}$ Bartolucci M, Businco L. Atopic dermatitis and food allergy in EuropePrevalence and risk factors. Allergy 1998; 53 (46 Suppl): 136-138

${ }^{5}$ Langeland T, Braathen LB, Borch M. Studies of atopic patch tests. Acta Derm Venerol 1989; 144 (Suppl. Stockh): 105-109

${ }^{6}$ Bohle B, Schwihla H, Hu HZ, Friedl-Hajek R, Sowka S, Ferreira F, Breiteneder H, Bruijnzeel-Koomen CA, de Weger RA, Mudde GC, Ebner C, Van Reijsen FC. Long-lived Th2 clones specific for seasonal and perennial allergens can be detected in blood and skin by their TCR-hypervariable regions. J Immunol 1998; 160: 2022 - 2027

7 Werfel T, Kapp A. Environmental and other major provocation factors in atopic dermatitis. Allergy 1998; 53: $731-739$

${ }^{8}$ Darsow U, Behrendt H, Ring J. Gramineae pollen as trigger factors of atopic eczema: Evaluation of diagnostic measures using the atopy patch test. Br J Dermatol 1997; 137: 201

${ }^{9}$ Darsow U, Vieluf D, Ring J. Evaluating the relevance of aeroallergen sensitization in atopic eczema with the atopy patch test: a randomised, double-blind multicenter study. J Am Acad Dermatol 1999; 40: 187-193

${ }^{10}$ Bruijnzeel PL, Kuijper PH, Kapp A, Warringa RA, Betz S, BruijnzeelKoomen CA. The involvement of eosinophils in the patch test reaction to aeroallergens in atopic dermatitis: Its relevance for the pathogenesis of atopic dermatitis. Clin Exp Allergy 1993; 23/2: 97-109

${ }^{11}$ Darsow U, Vieluf D, Ring J. The atopy patch test: An increased rate of reactivity in patients who have an air-exposed pattern of atopic eczema. Br J Dermatol 1996; 135: 182 - 186

12 Worm M, Henz BM. Molecular regulation of human IgE synthesis. J Mol Med 1997; 75: 440-447

${ }^{13}$ Kraft S, Wessendorf JH, Hanau D, Bieber T. Regulation of the high affinity receptor for IgE on human epidermal Langerhans cells. J Immunol 1998; $161: 1000-1006$

${ }^{14}$ Schaefer H, Zesch A, Stüttgen G. Skin Permeability. Berlin: Springer Verlag, 1982

${ }^{15}$ Kinet JP. Atopic allergy and other hypersensitivities. Curr Opin Immunol1999; 11: 603-605

${ }^{16}$ Schaefer H, Lademann J. The role of follicular penetration. A differential view. Skin Pharmacol Appl Skin Physiol 2001; 14 (Suppl 1): 23-27

17 Simon GA, Maibach HI. The pig as an experimental animal model of percutaneous permeation in man: Qualitative and quantitative observations - An overview. Skin Pharmacol Appl Skin Physiol 2000; 13: $229-234$

18 Weigmann HJ, Lademann J, Meffert H, Schaefer H, Sterry W. Determination of the horny layer profile by tape stripping in combination with optical spectroscopy in the visible range as a prerequisite to quantify percutaneous absorption. Skin Pharmacol Appl Skin Physiol 1999; 12: $34-45$

19 Ogiso T, Paku T, Iwaki M, Tanino T. Percutaneous penetration of fluorescein isothiocyanate-dextrans and the mechanism for enhancement effect of enhancers on the intercellular penetration. Biol Pharmacol Bull 1995; 18: $1566-1571$

20 Ogiso T, Paku T, Iwaki M, Tanino T. Mechanism of the enhancement effect of n-octyl-beta-D-thioglucoside on the transdermal penetration of fluorescein isothiocyanate-labeled dextrans and the molecular weight dependence of water-soluble penetrants through stripped skin. J Pharmacol Sci 1994; 83: 1676-1681 